\title{
ESTUDIO DE 130 AÑOS DE DEFUNCIONES POR SUICIDIO EN EL URUGUAY.1887- 2017.
}

\author{
STUDY OF 130 YEARS OF SUICIDE DEATHS \\ IN URUGUAY. 1887 - 2017
}

ESTUDO DE 130 ANOS DE MORTE POR SUICÍDIO NO URUGUAI. 1887-2017.

\section{Vignolo $\mathrm{J}^{1}$ Alegretti $\mathrm{M}^{2}$ Vacarezza $\mathrm{M}^{3}$ Álvarez $\mathrm{C},{ }^{4}$ Retamoso $\mathrm{E}^{5}$}

${ }^{1}$ Prof. Director Titular de Medicina Familiar y Comunitaria. Facultad de Medicina. UdelaR ${ }^{2}$ Prof. Adj. De Medicina Preventiva y Social. Facultad de Medicina. UdelaR ${ }^{3}$ Ex. Prof. Adj. Medicina Interna e Infectología. Facultad de Medicina. UdelaR

${ }^{4}$ Prof. Adj. Medicina Preventiva Social y Salud Ocupacional. Facultad de Medicina. UdelaR

${ }^{5}$ Educadora en Salud. Medicina Familiar y Comunitaria. Facultad de Medicina. Udelar.

Trabajo recibido: 17 de abril 2019.

Aprobado: 28 de junio

\section{Resumen}

El suicidio es una de las expresiones de violencia autoinfligidas, quecausa un gran impacto y daño en la familia, en la comunidad y en la sociedad. A nivel mundial se suicidan aproximadamente 800 mil personas por año y Uruguay es uno de los países con tasas de suicidio más elevadas. El objetivo de este estudio es determinar la tendencia de las muertes por suicidio en el Uruguay en el período comprendido entre 1887 y 2017. Se trata de un estudio de tipo descriptivo, del total de personas fallecidas por suicidio en el Uruguay, en el período de estudio. Para el análisis de tendencias se utilizó un modelo de regresión joinpoint, también se calcularon medias móviles y tendencia polinómica.Se observaque en estos 130 años,permanece un aumento significativo de la tasa $(p<0,05)$. Siendo el sexo masculino quien continúa presentando los valores más altos, conservando el aumento significativo de la tasa $(\mathrm{p}<0.05)$ durante todo el período.Uruguay continúa presentando una tasa estandarizada ubicada en el primer tercio de Países con las tasas más altas del mundo y de América. Se recomienda analizar, profundizar y realizar el permanente seguimiento del Plan Nacional de Prevención de Suicidio aprobado por la Autoridad Sanitaria.

Palabras Clave: Tasa de suicidio, Evolución histórica, Salud Pública, Masculinidades. 
Suicide is a type of self-inflicted violence which causes great impact and damage in the family, the community and the society. Worldwide, about 800,000 people commit suicide each year and Uruguay is one of the countries with the highest suicide rate. The objective of this study is to determine the tendency of suicide deaths in Uruguay in the period between 1887 and 2017. It is a descriptive study of people who committed suicide in Uruguay during the period studied. A joinpoint regression model was used to analyze trends; mobile averages and polynomial tendencies were also calculated. It was observed that in these 130 years there remains a significant rate increase $(p<0.05)$. Males continue presenting the highest values, and keeping the significant rate increase $(p<0.05)$ through the whole period. Uruguay still presents a standardized rate placed within the first third of countries with the highest rates in the world and America. It is advisable to analyze, intensify and do an ongoing follow up of the National Suicide Prevention Plan approved by the Health Authority.

Key Words: Suicide rate, Historical evolution, Public Health, masculinities.

Resumo:

O suicídio é uma das expressões de violência autoinfringida que causa grande impacto e abalo à família, à comunidade e à sociedade. No mundo, aproximadamente 800.000 pessoas cometem suicídio por ano e o Uruguai é um dos países com as maiores taxas de suicídio. O objetivo deste estudo é determinar a tendência de mortes por suicídio no Uruguai no período entre 1887 e 2017. Este é um estudo descritivo do número total de pessoas mortas por suicídio no Uruguai durante o período do estudo. Para a análise de tendência, utilizou-se um modelo de regressão joinpoint, calculando médias móveis e tendências polinomiais, observando-se que nesses 130 anos permanece um aumento significativo da taxa $(\mathrm{p}<0,05)$. Sendo o sexo masculino que continua apresentando os maiores valores, mantendo o acréscimo significativo da taxa $(\mathrm{p}<0,05)$ ao longo do período, o Uruguai continua apresentando uma taxa padronizada localizada no primeiro terço dos países com as maiores taxas do mundo e da América. Recomenda-se analisar, aprofundar e realizar o monitoramento permanente do Plano Nacional de Prevenção ao Suicídio, aprovado pela Autoridade de Saúde.

Palavras chave: Taxa de suicídio, Evolução histórica, Saúde pública, Masculinidades.

\section{Introducción}

El suicidio es una de las expresiones de violencia autoinfligidas, que revela un grado extremo de malestar, al punto que el individuo no percibe otra salida para solucionar los problemas o situaciones que vive. Causa un gran impacto y daño en la familia, en la comunidad y en la sociedad. Se trata de un fenómeno multifactorial, que se ha intentado de explicar por conductas y patologías personales. Pero estos aspectos y diagnósticos personales no pueden ser separados totalmente de las características tóxicas y violentas que posee nuestra sociedad en la actualidad. Estas características socio-culturales, en períodos de complejidad y globalizacióncomo el que vivimos, pueden llegar a agravar la realidad individual ya existente en ciudadanos que vivenestas situaciones de vulnerabilidad.(1)

No solo se debe tomar en cuenta los factores personales del individuo, al analizar este fenómeno, como lo son, los factores biológicos, genéticos, psicológicos y sociales (2); sino que estamos obligados a realizar un análisis más profundo de la sociedad y de la cultura, si deseamos que las medidas preventivas sean efectivas a la hora de aplicar planes nacionales sanitarios.

A nivel mundial se suicidan aproximadamente 800 mil personas por año, lo que supone 
una tasa anual de suicidio, ajustada según la edad, de 11,4 por cien mil habitantes $(15,0$ entre hombres y 8,0 entre mujeres), esto equivale auna muerte cada 40 segundos. Sin embargo, como el suicidio es un asunto sensible, incluso ilegal en algunos países, muy probablemente exista sub-notificación. Además, en los países con buenos datos de registro civil, el suicidio puede estar mal clasificado como muerte por accidente o por otra causa.(3)

A nivel mundial, los suicidios representan un 50\% de todas las muertes violentas registradas entre hombres y un $71 \%$ entre mujeres.(4)

Según datos del Institute For Health Metrics and Evaluation, se estima que a nivel mundial el 1,36\% de la carga global de morbimortalidad se debe a suicidio con un intervalo de confianza de 1,23-1,50\%. (IHME) (5) La comparación de las tasas entre los diferentes países continúa siendo difícil, dado los diferentes tipos de registros utilizados y por la permanencia de un importante subregistro en algunos de ellos. (6)

La tasa de mortalidad por suicidio es un indicador de la meta 3.4 de los Objetivos de Desarrollo Sostenible: «De aquí a 2030, reducir en un tercio la mortalidad prematura por enfermedades no transmisibles mediante su prevención y tratamiento, y promover la salud mental y el bienestar».(7)

Uruguay, continúa perteneciendo al grupo de países con tasas de suicidio más elevadas del mundo según los datos que hace referencia la OPS OMS, ocupando además el segundo o tercer lugar del continente Americano. (3)

Uruguay prácticamente ha completado la transición demográfica y epidemiológica, situación que se traduce en una mayor prevalencia de enfermedades no trasmisibles y en el aumento de los grupos de mayor edad. (8)

En este sentido, muestra que las características son similares a la de los países desarrollados. Uruguay presentó en el año 2017 una expectativa de vida de 77 años 74 para el hombre y 81 para la mujer), una tasa global de fecundidad de 1,81 (9); una tasa bruta de mortalidad general de 9,50 por mil(10)y una mortalidad infantil de 6,5 por mil.(11, 12)

Entre los factores de riesgo más importantes encontrados en la literatura para la mortalidad por suicidio se señalan: la edad, el sexo, los factores biológicos, psiquiátricos, sociales y ambientales, así como los factores vinculados con la historia personal del individuo.(13) Los estudios realizados a nivel internacional y nacional muestran una prevalencia de problemas mentales en personas que presentaron muertes por suicidio de entre 70 a 100\%. En general en las diversas autopsias psicológicas se encuentra un claro predominio de los procesos depresivos. Acontecimientos de la vida pueden actuar como factores desencadenantes: pérdida de seres queridos, depresión, conflictos laborales, desempleo, soledad, maltrato, abuso sexual en la niñez, relación de pareja inestable o soledad, aislamiento social, fácil acceso a métodos suicidas, problemas económicos sociales etc. $(13,14)$

El objetivo de este estudio fue determinar la tendencia de las muertes por suicidio en el Uruguay en el período comprendido entre 1887-2017 a 130 años de haber comenzado el registro del fenómeno en el país.

\section{Material y métodos}

Se trata de un estudio de tipo descriptivo del total de personas fallecidas por suicidio, en la República Oriental del Uruguay, en el período 1887-2017.

La información sobre población entre los 1895 a 1949, fue tomada de las proyecciones de población del programa de población de la Facultad de Ciencias Sociales de Adela Pellegrino (2000). Entre 1950 y el año 2000 se utilizaron las estimaciones y proyecciones de la población por sexo y edad de CEPAL-CELADE-INE (1998). Del año 2001 en adelante se utilizaron las proyecciones de población total por sexo y edad 1996-2025 Revisión 2013 del Instituto Nacional de Estadísticas.

Las defunciones entre 1887 y 1949 tuvieron como fuente de información la Serie Histórica de Mortalidad en el Uruguay por causa y sexo desde fines del Siglo XIX, 1897-1996. De 
los años 1887 a 1896 y de los años 1898, 1899, 1902, 1908, 1922, 1926,1927 y 1949 fueron tomadas de la Facultad de Ciencias Sociales del Programa de Población de Adela Pellegrino y del correspondiente Censo de Población. Entre 1950 y 1996, de la serie histórica de Mortalidad en el Uruguay, MSP/FISS. Entre 1997 y el año 2000, del Departamento de Estadística de la Dirección General de la Salud del Ministerio de Salud Pública (DIGESAMSP). Entre 2001 y 2017 fue tomada las series de tiempo referidas a las defunciones del Uruguay del período 1999-2017 (MSP/Estadísticas Vitales).(11)

Para el análisis de tendencias se utilizó un modelo de regresión joinpoint mediante el programa informático proporcionado por el Surveillance Research Program del National Cancer Institute de EE. UU.(15)

El modelo de regresión joinpoint es uno de los métodos más empleados para estimar los cambios en la tendencia de tasas de mortalidad. (16) Este modelo permite identificar el momento en que se producen cambios significativos de la tendencia de una serie, y estima la magnitud del aumento o el descenso observado en cada intervalo o período de tiempo. De esta manera se identifican los años que componen cada período, así como el porcentaje de cambio anual (PCA) y sus intervalos de confianza.

Para la estimación del modelo se utilizó el logaritmo natural de las tasas de mortalidad, se fijó en 4 el mínimo número de datos en la tendencia lineal y se buscó un máximo de 3 puntos de inflexión.

Para la comparación de las series según sexo, se utilizaron medias móviles y cálculo de la tendencia polinómica, utilizando Excel. La línea de tendencia polinómica o curvilínea utiliza la ecuación polinómica: $\mathrm{g}=\mathrm{b}+\mathrm{c} 1 \mathrm{x}+\mathrm{c} 2 \mathrm{x} 2+\mathrm{c} 3 \mathrm{x} 3 \ldots+\mathrm{c} 6 \mathrm{x}$

Se utilizó la prueba de hipótesis z para la diferencia de tasa.

\section{Resultados}

La evolución de las tasas específicas de mortalidad por suicidio en 130 años de historia, en el Uruguay (1887-2017)muestra un comportamiento irregular, con un rango entre 4,04 por cien mil en 1889 a 20,89 por cien mil en 2002.

En la tabla $\mathrm{N}^{\mathrm{o}} 1$ se presentan los resultados del modelo, que identifica cuatro segmentos lineales (períodos) y tres puntos de unión (1894, 1934 y 1981), de los cuales dos años fueron puntos de inflexión (1934 y 1981) en los que cambió el sentido de la línea de tendencia.

Tabla $\mathbf{N}^{0}$ 1: Resumen del modelo de regresión joinpoint para tasa de suicidio. Uruguay $1887-2017$

\begin{tabular}{|c|c|c|c|c|c|c|c|}
\hline Segmento & Añoinicial & Año final & $\begin{array}{c}\text { Variación } \\
\text { anual } \\
\text { (PCA) }\end{array}$ & IC inferior & $\begin{array}{c}\text { IC } \\
\text { superior }\end{array}$ & $\begin{array}{c}\text { Test } \\
\text { estadístico } \\
(t)\end{array}$ & Valor $p$ \\
\hline 1 & 1887 & 1894 & $9,0 *^{*}$ & $2,8 \%$ & $15,7 \%$ & 2,9 & $<0,05$ \\
\hline 2 & 1894 & 1934 & $1,4 \%^{*}$ & $1,0 \%$ & $1,9 \%$ & 6,3 & $<0,05$ \\
\hline 3 & 1934 & 1981 & $-0,9 \%^{*}$ & $-1,3 \%$ & $-0,6 \%$ & $-5,2$ & $<0,05$ \\
\hline 4 & 1981 & 2017 & $2,1 \% *$ & $1,6 \%$ & $2,6 \%$ & 8,1 & $<0,05$ \\
\hline
\end{tabular}

Si se analiza el comportamiento de la tasa de suicidio utilizando el modelo de regresión (Gráfico $\mathrm{N}^{\circ}$ 1) se observa un primer período de rápido acenso situado entre 1887 y 1894, donde hubo un aumento estadísticamente significativo en la tasa de suicidio, con un PCA de $9,01 \%$, pasando de 5,2 por cien mil a 8,6 por cien mil.

Un segundo período de ascenso se sitúa entre 1894 y 1934, donde también hubo un aumento estadísticamente significativo de la tasa de suicidio, pero de menor intensidad que 
en el período anterior. El PCA fue 1,42\%, pasando de una tasa de 8,6 por cien milen 1894 a 19,2 por cien mil en 1934. En este período encontramos un primer máximo en 1900, con una tasa de 16,72 por cien mil, un segundo máximo con una tasa de 14,83 por cien mil en 1917 y un tercero en 1934 con la tasa más alta del sigo XX (19,2 por cien mil). Si se compara la tasa de suicidio en 1887 con la de 1934 observamos un crecimiento de $370 \%$, diferencia estadísticamente significativa $(\mathrm{p}<0,05)$

En 1934 encontramos el primer punto de inflexión de la serie, comenzando eltercer período, que muestra descenso estadísticamente significativo en la tasa de suicidio con un PCA de $-0.92 \%$, llegando a una tasa de 9,1 por cien mil en 1981 .

Luego aparece el segundo punto de inflexión de la serie, comenzando un cuarto período entre 1981 y 2017 con ascenso estadísticamente significativo y casi continuo. Durante este último período, en el año 2002, la tasa de suicidio alcanza el 20,89 por cien mil, siendo de esta manera la más alta de la historia. El PCA en este período fue $2,1 \%$ y es estadísticamente significativo $(\mathrm{p}<0,05)$.

Si se compara la tasa de suicidio en 1981 (11,18 por cien mil) con la de 2017 (19,70 por cien mil) observamos un aumento de 49\%, $(\mathrm{p}<0,05)$.

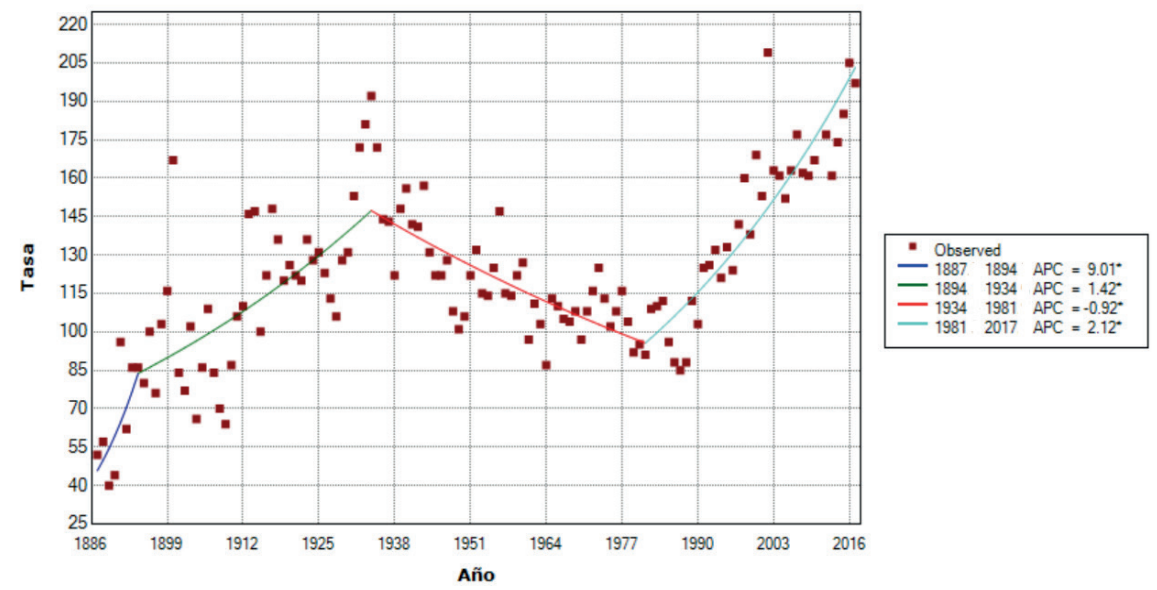

- Indicates that the Annual Percent Change (APC) is significantly different from zero at the alpha $=0.05$ level.

Gráfico $\mathbf{N}^{\mathbf{0}}$ 1: Tasa de Suicidio según año y modelo de regresión Uruguay 1887-2017.

En relación al sexo se observa la mayor prevalencia de la tasa masculina, con respecto a la tasa general y a la femenina a lo largo de todo el período estudiado (Gráfico $\mathrm{N}^{\circ} 2$ )

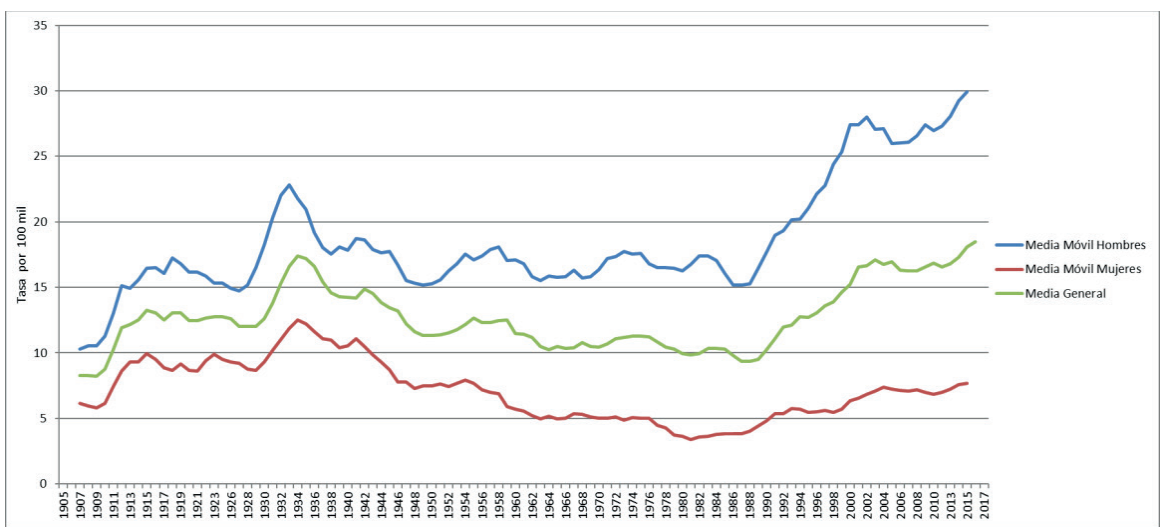

Gráfico $\mathbf{N}^{0}$ 2: Distribución de las medias móviles de suicidio por sexo, según año. Uruguay $1905-2017$ 
El análisis del promedio móvil, (Gráfico $\mathrm{N}^{\circ}$ 2) y de la tendenciapolinómica, (Gráfico $\mathrm{N}^{\circ}$ 3) para el sexo masculino y femenino en el período comprendido entre 1905 y 2017 (no es igual al período de la tasa general debido a que se contó con datos de sexo a partir de 1905) muestra una brecha que separa a la tasa masculina de la femenina, una mayor prevalencia de la tasa masculina sobre la tasa general y de la femenina.

La tasa global y la tasa masculina presentaron las cifras más elevadas en la historia en el año 2002, sin embargo si se considera la media móvil, la tasa más elevada corresponde a los últimos tres años.

Se observa a lo largo del tiempo un aumento de la brecha entre ambos sexos. En 1905 la razón masculino /femenina fue de 1,6, en 1934 de 1,8; en 1981 llegó al valor máximo de 5,4 para luego disminuir y oscilar en torno a 4 hasta el 2017 (que tuvo una razón masculino/femenina de 4,4).

En consecuencia la brecha crece a principios de la década del $80^{\prime}$ fundamentalmente a expensas de una disminución de los suicidios femeninos y el mantenimiento de la tasa masculina.(17)

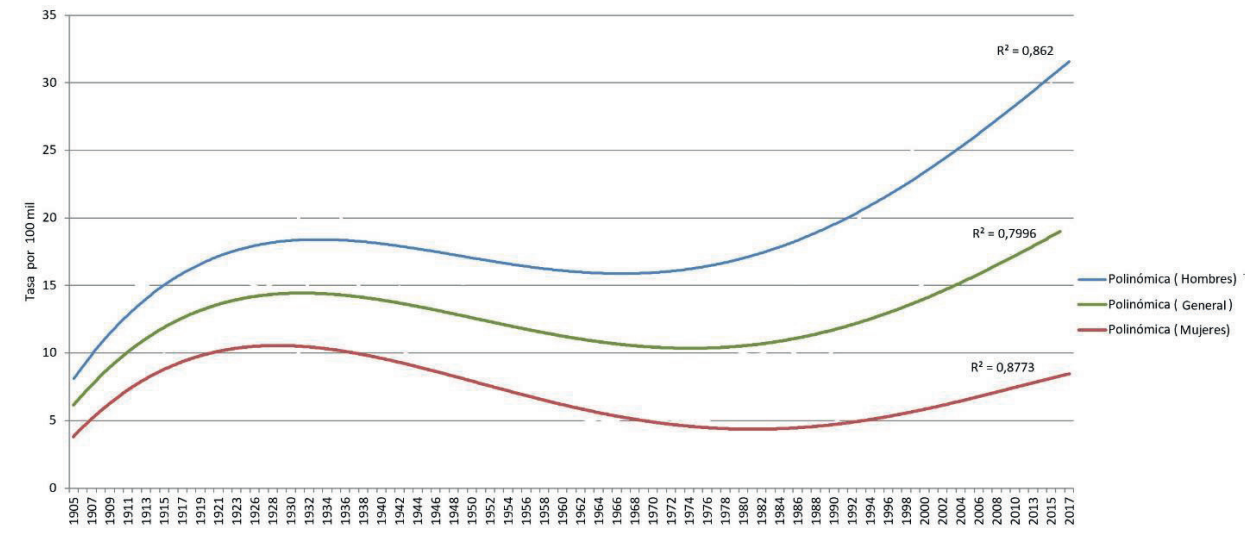

Gráfico $\mathbf{N}^{0}$ 3: Distribución de la tendencia polinómica de las tasas de suicidio por sexo según año. Uruguay 1905-2017

El comportamiento de la tasa masculina (Gráfico $\mathrm{N}^{\circ} 2$ ) estudiada a través del promedio móvil, nos muestra períodos claramente diferenciados: Un primer período, que muestra el aumento de la tasa entre 1905 y 1934 pasando de 10,74 a 24,51 por cien mil, un segundo período estacionario entre 1936 y 1988, con tasas de 15,75 y 14,29 por cien mil respectivamente, un tercer período de aumento entre 1989 y el año 2017 llegando a la tasa de mayor valor del período en el 2002.

En la tasa femenina (Gráfico $\mathrm{N}^{\circ}$ 2) podemos distinguir un primer período, entre $1905 \mathrm{y}$ 1934 de ascenso, donde la tasa pasa de 6,37 a 13,70 por cien mil respectivamente, un segundo período, entre 1937 y 1985 de descenso, con tasas entre 10,23 a 3,38 por cien mil respectivamente, un tercer período de ascenso con oscilaciones hasta 2017 (tasas de 3,72 a 7,44$)$

\section{Discusión}

La tasa de suicidio ha aumentado en los 130 años considerados en forma estadísticamente significativa, presentó grandes elevaciones dada por los años 1934 y 2002.

El aumento de la tasa de suicidio en el siglo XX en particular, en el año 1934, el pico mayor de ese siglo se desarrolla en un contexto de crisis económica ocurrida en 1929 y de ruptura institucional en 1933, en donde el ex Presidente de la República Baltasar Brum en señal de protesta se suicida utilizando como método de disparo un arma de fuego. $(17,18)$ 
La crisis económica que tuvo origen en los Estados Unidos sin duda marco este período. $(20,21,22)$

Esta situación económica llevó a una inestabilidad política que concluyó con el golpe de estado, con un desequilibrio económico que afecto al País de manera general. $(19,20)$

Durante la gran crisis económica del 2002, que debilitó al sector financiero se constituyó un proceso de insolvencia financiera que afectó a más de la mitad de la banca comercial, y produjo la desaparición de algunas empresas financieras más emblemáticas y el virtual colapso del sistema económico del País. Es en este período de crisis económica-social, donde se da el mayor valor de la tasa de suicidio en la historia del País.(18)

Se señalan en otros estudios los cambios socio-económicos, en cualquier dirección como uno de los factores en una problemática multicausal, que contribuyen elevar las tasas. (23, $24,25)$ Se destaca asimismo, que no solo los cambios contribuyen a elevarlas sino también, el entorno y las condiciones de vida son determinantes. (24)La violencia no es más que una forma de expresión de todos los aspectos anteriormente señalados. (26)

Según Durkheim, los hombres son los que más se suicidan; se podría decir que el suicidio es una inclinación masculina. (27)Si bien el suicidio predomina en el sexo masculino, la relación hombre/mujer varía con los distintos países. $(28,29,30)$

A nivel mundial la OMS (2001) señala una razón de 3,5 a 1 entre ambos sexos En diversos países de Europa: Inglaterra y Gales (31, 32)Austria (27); Países Bálticos (33, 34, 35)Italia (36) se reportaron tasas de suicidio superiores en el sexo masculino. La razón hombre / mujer paso de 3,1 en 1979 a 4,5 en 1994 en los EEUU y más de los 70\% de los suicidios fueron cometidos por hombres.(37,38) En Chile entre 1989 y 1995 se encontró una relación hombre/mujer de 4,8 a 1.(39) Robertt estudió en el Uruguay el período 1963-1993, encontrando el aumento de la razón hombre/mujer en el mismo. De acuerdo a lo manifestado por este autor, se observa, como los hombres fueron más sensibles a los movimientos de conmoción política y económica (obsérvese los movimientos de la curva masculina que son anteriores en el tiempo). (40)

Dados estos resultados, se hace referencia conceptual a la temática de género y en particular la construcción de las masculinidades ya que se han constituido en un campo de reciente referencia conceptual. Sólo si se integran la temática, se podrá trabajar para lograr un cambio social a largo plazo sobre la equidad de género, disminuyendo la inequidad en salud mental en los determinantes estructurales, que participan en la construcción de lo masculino. No se trata de realizar un análisis exclusivo, sino por el contrario, incorporar dicha perspectiva al análisis general sobre suicidio, transversalizando la categoría de género, para aproximarnos en futuros estudios, a los determinantes socio-culturales que inciden en la construcción de las masculinidades.

La formación en un sistema de creencias y valores va educando a los hombres y mujeres a integrar la violencia en las formas sociales admitidas para enfrentar los conflictos. Se destaca entonces que el género masculino, es ese común denominador en mayor número de fallecimientos por siniestralidad vial y por suicidios.

Dada esta realidad se puede preguntar. ¿Qué mensaje reciben los varones para la trasgresión de reglas que los desvinculan de los cuidados personales y de la vida?

¿A qué aislamiento social y emocional se ven sometidos los hombres durante su vida, que la expresión de búsqueda de la masculinidad es el riesgo, la transgresión o poner fin a su vida si no logran colmar sus expectativas?

¿Cuál es la construcción social y el imaginario social de esa masculinidad que vincula casi en forma permanente el peligro al cotidiano?

Bonino plantea que la patología mental masculina, es necesario que aparezca como tal y sea motivo de estudio y tratamiento específico. Los malestares masculinos y la predominancia en algunos de ellos no se caracterizan como tales en ámbitos sanitarios, lo que lleva a la invisibilización de los mismos. (41)

Por el contrario, cuándo se puede disociar sus construcciones teóricas, se coloca como ele- 
mento insustituible no sólo para comprender el malestar de género de hombres y mujeres, sino también para dar lugar a la conceptualización de las profundas transformaciones que se encuentran hoy en proceso de la subjetividad de hombres y mujeres. (42)

\section{Conclusión}

A lo largo de los 130 años de estudio existieron variaciones significativas en las tasas de suicidio, existiendo cuatro períodos diferenciados, los dos primeros de aumento (con diferente velocidad), el tercero de descenso y el cuarto con un incremento que elevó la tasa a los valores más altos de la historia. Los aumentos bruscos coincidieron con etapas de conmoción social y económica. El análisis por sexo, muestra que la tasa de suicidio sigue siendo mayor en los hombres.

Podemos afirmar que en el Uruguay el suicidio, por su magnitud e impacto en la población constituye un problema de salud pública.

\section{RECOMENDACIONES:}

Se recomienda analizar, profundizar y realizar el permanente seguimiento del Plan Nacional de Prevención de Suicidio publicado para el periodo 2011-2015 por la Autoridad Sanitaria. Estimamos que el Plan Nacional de Suicido debería profundizar en la capacitación y fortalecimiento a nivel de los profesionales del primer nivel de atención, elementos sustanciales para su prevención y seguimiento.

\section{Bibliografía}

1. Vignolo J, Henderson E, Vacarezza M, Álvarez C, Alegretti M, Sosa A. Análisis de 123 años de muertes por suicidio en el Uruguay, 1887-2010. Revista de Salud Pública, (XVII) 1:8-18, abril 2013. Argentina.

2. Paparambordda MC, Vignolo JC, González S. Estudio epidemiológico del suicidio en el Uruguay en el contexto de las muertes violentas 1963-1999. Montevideo., 1999;1-28 (Documento de circulación Interna ,MSP

3. Vignolo JC. Estudio del Suicidio en el Uruguay. Período 1887-2000 y Análisis epidemiológico del año 2000. Tesis de Maestría en Epidemiología. Departamento de Medicina Preventiva y Social, 2004.

4. WHO Prevención del suicidio un imperativo global. 2014 https://www.who.int/ mental_health/suicide-prevention/world_report_2014/es/. [Consultado 20 Marzo 2019]

5. Institute For Health Metrics and Evaluation. Data Visualizations. Disponible en: http://www.healthdata.org/results/data-visualizations. [Consultado 5 Abril 2019]

6. OMS. Informe sobre la salud del mundo 2001. Salud Mental: Nuevos conocimientos, nuevas esperanzas. Francia 2001.

7. Fuente WHO Suicidio. Datos y Cifras. Disponible en https://www.who.int/es/newsroom/fact-sheets/detail/suicide. [Consultado 9 Abril 2019]

8. MSP. Mortalidad en el Uruguay año 2010. Disponible en http://WWW.msp.gub.uy/ andocasociado.aspx?6490,23187. [Consultado 20 Marzo 2019]

9. Instituto Nacional de Estadísticas. Indicadores Demográficos, Series Históricas. Disponible en: http://www.ine.gub.uy/web/guest/indicadores-demograficos1. [Consultado 9 Marzo 2019]

10. INE. Proyecciones de población total y departamental por sexo y edad 19962025. Disponible en: http:/WWW.ine.gub.uy/socio-demograficos/proyecciones2008.asp [Consultado 3 Abril 2019]

11. MSP Estadísticas vitales. Disponible en: https://uins.msp.gub.uy/. [Consultado 5 Abril 2019] 
12. MSP. Mortalidad infantil en el Uruguay 2011. Disponible en

WWW.msp.gub.uy/andocasociado.aspx?6487,23703[Consultado 5 Abril 2019]

13. OPS/OMS. Informe Mundial sobre violencia y la salud. Publicación Científica y Técnica. Washington D.C.2003; 588.

14. Lucero A, Rossana K, Díaz N, Villalba L. Caracterización clínica y epidemiológica de los suicidios en Montevideo y de los intentos de autoeliminación (IAE) en el Hospital de Clínicas en el período abril 2000-abril 2001: el método de la autopsia psicológica. Rev. Psiquiatr. Urug; 67(1):5-20, ago.2003

15. (National Cancer Institute. Joinpoint Regression Program [Soft-ware]. Versión 4.0.4. StatisticalResearch and A pplications,NationalCancerInstitute, [Consultado 9 de Marzo 2019].Disponible en: http://srab.cancer.gov/joinpoin).

16. Vidal C, Hoffmeister L, Biagini L. Tendencia de la mortalidad por cáncer de cuello uterino en Chile: aplicación de modelos de regresión joinpoint. RevPanam Salud Pública. 2013; 33(6):407-13.

17. Vignolo J, Paparamborda MC, Murillo N, Hernández S, Pérez C, Méndez A. Análisis epidemiológico de los fallecimientos por suicidio, Uruguay Año 2000. Revista de Salud Pública, (XIII) 1:6-17, jun. 2009. Argentina.

18. Academia Nacional de Economía. Contribución a la Historia económica del Uruguay. Montevideo, 1984

19. Universidad de la república: Facultad de Ciencias Económicas y de AdministraciónInstituto de Economía. El Proceso económico del Uruguay: contribución al estudio de su evolución y perspectivas. Dpto. de Publicaciones de la Universidad de la República. Montevideo, 1969.

20. Nahum B. Manual de Historia del Uruguay. Tomo II: 1903-2000. EBO, $11^{\text {a }}$ ed, Montevideo. 2003.

21. Nahum B. Breve Historia del Uruguay independiente. EBO, Montevideo. 2003

22. Dajas F. Alta tasa de suicidio en Uruguay. Consideraciones a partir de un estudio epidemiológico. Rev. Méd. Uruguay. 1990; (6): 203-15.

23. Almendras I. Método EBE, estrategias para el "Bien - Estar" Manual de psicoeducación para trabajadores de la salud y educadores. 1a edición. Ediciones del Mileno, Montevideo. Mayo 2002.

24. Almendras I. ¿Bien estar o depresión? Guía para estar bien. $1^{\text {a }}$ Edición. Ediciones del Milenio, Montevideo. Mayo 2001.

25. González Rea J. Estudio epidemiológico de las muertes en el Uruguay. Monografía de Posgrado en Psiquiatría. Archivo de Monografías de Posgrado. Biblioteca de la Facultad de Medicina, Montevideo, Uruguay .1998.

26. Durkheim E. El suicidio. El suicidio de sociología (1987). Bs.As. Schapire Ed. 1952; 7-315.

27. OMS. Preventing suicide: a resource for general phsicians. WHO/MNH/ MBD/00.1.Geneva 2000;1-18

28. Crome P. The toxicity of drugs used for suicide. Acta PsychiatryScand. 1993; (371) 33-37

29. Crumley FE. Substance abuse and adolescent suicidal behaviour. JAMA. 1990263 (22): 3051-1056

30. McClure G. Trends in suicide rare for England an Wales, 1975-1980. British Journal of Psychiatry. 1984; (144): 119-126

31. (1987) suicide in England and Wales, 1975-1984. British Journal of Psychiatry. 150:309-314.

32. (194) Suicide in children and adolscents in England an Wales, 1960-1990. British Journal of Psychiatry, 165:510-514.

33. Mc. Clure G. Changes in suicide in England an Wales, 1960-1997. British JournalPsychiatry. 2000; (176):64-7 
34. Retamal C, Humpreys D. Occurrence of suicide and seasonal variation. Rev. SaúdePública. Wasserman D, Varnik K, Eklund G. Male suicides and alcohol consumption in the former Wasserman D, Varniik A. Increase in suicide among men in the baltic countries. Lancet. 1994; (343):1504-1505.

35. Editorial Suicide: Risk factors and the public healt. American Journal of Public Health. 1993; 83(2):1712.

36. Móscicki E. Identification of suicides risk factors using epidemiologic studies. In: ThePsychiatricClinics of North America. Suicide. 2000; 449-514.

37. Singh GK, Kochanek KD, MAcDorman MF. Advance Report of final Mortality Statistics, 1994. Monthly Vital Statistics Report). Hyattsville, MD, National Center for Health Statistic. 1996; 3 (45).

38. Méndez V, Opgaard J, Escalier A, Segundo C, Reyes J, ZamoraL, Hurtado L, Olivares Y. Epidemiología del suicidio en la segunda región de Chile/Epidemiology of suicide al thesecond región of Chile. Rev. Chil. Neuropsiquiatr. 1997; 35(4):465-72

39. Robertt P. El suicidio en Uruguay. ISNN- 0187- 3148. 1999;(6):66-78

40. Vignolo J, Alegretti M, Vacarezza M, Álvarez C, Evolución de las muertes por suicidio en el Uruguay a nivel Departamental período 2001-2010. Revista de Salud Pública, (XIX) 1:6-13, jul. 2015.

41. Bonino L. Varones, Género y Salud Mental. Desconstruyendo la "Normalidad Masculina". Publicado en Segarra M y Carabí A. (eds) (2000) Nuevas masculinidades. Barcelona: Icaria.

42. Fernández, AM. La diferencia como problema: Género y psicoanálisis. Nómadas (Col) [Internet]. 1997;(6). Recuperado de: https://www.redalyc.org/ articulo.oa?id=105118999003. [Consultado 3 Abril 2019] 